\title{
Defective Protein Catabolism in Atherosclerotic Vascular Inflammation
}

\author{
Takuro Miyazaki* and Akira Miyazaki \\ Department of Biochemistry, School of Medicine, Showa University, Tokyo, Japan
}

Vascular inflammation in atheroprone vessels propagates throughout the arterial tree in dyslipidemic patients, thereby accelerating atherosclerotic progression. To elucidate the mechanism of vascular inflammation, most previous studies have focused on inflammation-related signals that are sent in response to vasoactive stimuli. However, it is also important to understand how normal blood vessels become defective and start degenerating. Growing evidence suggests that major protein catabolism pathways, including the ubiquitin-proteasome, autophagy, and calpain systems, are disturbed in atheroprone vessels and contribute to the pathogenesis of atherosclerosis. Indeed, dysregulation

OPEN ACCESS

Edited by:

Masanori Aikawa,

Harvard Medical School,

United States

Reviewed by:

Luigi Michele Pavone,

University of Naples Federico II, Italy Katsuya Hirano,

Kagawa University, Japan

*Correspondence:

Takuro Miyazak

taku@pharm.showa-u.ac.jp

Specialty section: This article was submitted to

Atherosclerosis and

Vascular Medicine,

a section of the journal

Frontiers in Cardiovascular Medicine

Received: 20 September 2017

Accepted: 23 November 2017

Published: 07 December 2017

Citation:

Miyazaki T and Miyazaki A (2017) Defective Protein Catabolism in Atherosclerotic Vascular Inflammation.

Front. Cardiovasc. Med. 4:79. doi: 10.3389/fcrm.2017.00079 of ubiquitin-proteasome pathways results in the accumulation of defective proteins in blood vessels, leading to vascular endothelial dysfunction and apoptosis in affected cells. Impaired autophagy-lysosomal degradation affects smooth muscle cell transformation and proliferation, as well as endothelial integrity and phagocytic clearance of cellular corpses. Dysregulation of the calpain system confers proatherogenic properties to endothelial cells, smooth muscle cells, and macrophages. In this review article, we will discuss the current information available on defective protein catabolism in atheroprone vessels and its potential interrelation with inflammation-related signals.

Keywords: calpain, autophagy, ubiquitin proteasome, nitric oxide, inflammasome, mechanotransduction, apoptosis, efferocytosis

\section{INTRODUCTION}

Atherosclerosis is a chronic inflammatory disease accompanied by the intimal thickening of systemic arterial walls $(1,2)$. Rupture of vulnerable plaques as well as thrombotic/embolic occlusion and arterial narrowing can be causal of lethal ischemic disease, including acute coronary syndromes/ myocardial infarction (3). Regarding the pathogenic cues that contribute to atherosclerosis, the majority of previous atherosclerosis studies support the hypothesis that atherosclerosis is driven through reactive oxygen species (ROS)-mediated oxidative stress, which leads to the induction of numerous inflammatory elements, such as adhesion molecules in vascular endothelial cells (ECs) and proinflammatory cytokines via redox-sensitive transcription factors (2). During prolonged vascular inflammation, degenerative insults, such as increased numbers of apoptotic cells, remodeling of the extracellular matrix (ECM), breakdown of elastic lamella, and the dysfunction of ECs, emerge in atheroprone vessels and accelerate atherosclerosis-related complications (2). Although earlier clinical trials investigated the efficacy of antioxidants, these agents failed to reverse cardiovascular death (4); in contrast, they were effective in ameliorating acute inflammation, including the acute phase of 
stroke (5). Therefore, in addition to the mechanisms underlying acute inflammatory insults, it is important to understand how blood vessels shift toward degenerative status in chronic vascular disease. In this regard, accumulating evidence indicates that defects in protein catabolism systems, which consist of a variety of intracellular proteases, critically contribute to inflammatory vascular degeneration. Autophagy, an essential intracellular process mediated by the lysosomal degradation of cytoplasmic components, is detectable in every tissue and mediates the nutrient turnover, particularly in cells under starving conditions. Although this system is involved in sustaining cellular metabolism, homeostasis, and survival (6), autophagic flux was detected in atheroprone vessels (7). Furthermore, the ubiquitin-proteasome system, which acts as a threshold machinery for protein catabolism (8), is regulated through proinflammatory cytokines such as interferon- $\gamma$ (9), oxidized low-density lipoprotein (LDL) (10), and oxidized cholesterol (11). In addition to their roles in protein degradation, several classes of proteases transduce cellular signaling through their ability to process target proteins in stress-inducing environments. Indeed, calpain, a $\mathrm{Ca}^{2+}$-dependent intracellular cysteine protease, is activated in response to several classes of cytokines, growth factors, lysophospholipids, and physical stresses (12), thereby participating in degenerative vascular disorders $(13,14)$. In this review, we summarize the recent achievements in the proinflammatory and proatherogenic defects of these protein catabolism pathways. In addition, we will discuss anti-atherosclerosis strategies that target defective protein catabolism.

\section{DEFECTIVE PROTEIN CATABOLISM UNDERLIES ATHEROSCLEROSIS}

\section{Autophagy}

Autophagy is a self-degenerative process that participates in organelle turnover, and the recycling of cytoplasmic components as well as protein degradation in response to extracellular stresses. Currently, autophagy can be categorized into three classes (6). Microautophagy is a non-selective lysosomal process describing the direct engulfment of cytoplasmic cargos by lysosomes. This process is accomplished by the inward invagination of cargos into the lysosomal membrane. In contrast, chaperone-mediated autophagy enables the selective degradation of cytoplasmic proteins by recognizing chaperone proteins. During this process, lysosomal-associated membrane protein type $2 \mathrm{~A}$ on the lysosomal membrane recognizes the target proteins via their chaperone (e.g., heat shock cognate protein of $70 \mathrm{kDa}$ ), allowing internalization of the target protein into lysosomes. Macroautophagy is a process whereby cytoplasmic components are degraded by lysosomes, and it is accompanied by the formation of autophagosomes. These autophagosomes are cytosolic double-layered membrane vesicles, in which cytoplasmic components are separated from the cytoplasmic environment, and which finally fuse with a lysosome where lysosomal digestion occurs. One of the key regulators of autophagy is the kinase mammalian target of rapamycin (mTOR), which negatively regulates autophagy (6). Autophagosome formation is mediated by Atg genes, which is regulated by Atg12-Atg5-Atg16L1 and LC3-PE (Atg8 homolog) complexes (6). It was reported that nutrient supply by macroautophagic protein catabolism sustained anabolic reactions to generate macromolecules, such as nucleic acids, proteins, and organelles. As a result, the nutrient supply can exert a prosurvival mechanism, particularly under starving conditions (15). In contrast, the overactivation of autophagic systems elicits cell death, which may be mediated by a process morphologically distinct from apoptosis (16). The contribution of macroautophagy to tumorigenesis, infection, diabetes, and cardiovascular diseases was described previously $(6,7,15)$. In vascular systems, macroautophagy is detectable in macrophages in atherosclerotic lesions as well as in vascular-resident cells, including ECs and vascular smooth muscle cells (VSMCs), where it participates in physiologic and pathogenic responses (6).

Razani et al. reported that macroautophagy was associated with the pathogenesis of atherosclerosis (17). The expression of p62 protein, but not of p62 mRNA, was potentiated in lesional macrophages suggesting insufficient autophagy in these cells. Macrophage-specific deficiency of Atg5 accelerated the development of atherosclerosis in $A p o e^{-/-}$mice by insufficient autophagy in cells. Furthermore, Liao et al. also reported abnormal expression patterns of LC3 and the presence of p62-positive cells in atheromas in atherogenic mice, indicating impaired autophagy in the lesions (18). Accordingly, macrophage-specific Atg5 deficiency augmented the expansion of lesional necrotic cores and progression of atherosclerosis. Therefore, insufficient autophagy might decrease the stability of atherosclerotic plaques as well as its pathogenic implications.

\section{Ubiquitin-Proteasome System}

The ubiquitin proteasomal systems are part of the central protein catabolism machinery in cells and are involved in the degradation of misfolded proteins, as well as normal protein degradation. These systems are also implicated in cellular events, including antigen presentation in immune cells (19) and the cell cycle (20). The polyubiquitination of target molecules triggers proteasomal degradation, which is driven by $\mathrm{E} 1 \mathrm{ubiquitin}$-activating enzymes, E2 ubiquitin-conjugating enzymes, and E3 ubiquitin-protein ligases (21). Following the ubiquitination process, ubiquitinated target proteins are recognized by proteasomal complexes and are proteolytically degraded into peptides. In this process, the $26 \mathrm{~S}$ proteasome comprising regulatory particle $19 \mathrm{~S}$ and core particle 20S, has a crucial role in the proteasomal systems (9). Similar to autophagic systems, dysfunctional proteasomes can cause degenerative diseases, such as Alzheimer's disease (22), Parkinson's disease (22), Huntington's disease (22), and atherosclerosis (23, 24). An atherosclerosis study in high fat diet-fed pigs identified that proteasomal activity was elevated in coronary arteries during the progression of atherosclerosis (25). However, in a human atherosclerosis study, the carotid atherosclerotic lesions in subjects complicated with transient ischemic attack or stroke exhibited lower proteasomal activity compared with that in asymptomatic subjects (26). These studies suggest that proteasomal activity declines in advanced atherosclerotic plaques, whereas it is sustained in mild lesions. In addition to these observations, animal experiments with pharmacological proteasomal intervention 
have been performed. Herrmann et al. examined the long-term administration of the proteasome inhibitor MLN-273 in high cholesterol diet-fed pigs and identified the elevation of oxidative stress and exacerbation of atherosclerosis (25). Conversely, Wilck et al. reported that a moderate dose of bortezomib (also known as PS-341) led to partial proteasomal inhibition in high cholesterol diet-fed $\mathrm{Ldll}^{-/-}$mice, which reduced oxidative stress and NADPH oxidase 4 expression levels in the aortae, thereby suppressing atherosclerosis (27). Therefore, it hardly defines pathophysiologic roles of proteasomal systems in atherosclerosis. It is well known that macrophage cytotoxicity is prominent in advanced atheromas, while vascular inflammation is sustained throughout moderate-to-advanced atherosclerotic lesions (1). Thus, it is suspected that the contribution of proteasomal systems to the pathogenesis of atherosclerosis is substantially dependent upon the stage of the disease.

\section{Calpain Systems}

The calpain family comprises $\mathrm{Ca}^{2+}$-dependent intracellular proteases (28-30). These proteolytic systems are unique because calpains modulate their substrates through limited proteolytic cleavage in addition to proteolytic degradation. The calpain system comprises the endogenous inhibitor calpastatin as well as 15 homologs of the catalytic subunits, and 2 regulatory subunit homologs in mammals (28-30). Calpain catalytic subunits are categorized into conventional and unconventional subtypes (28-30). The conventional subtype consists of two isozymes, calpain-1 and calpain-2, which are ubiquitously expressed in vertebrates (28-30). These are heterodimers of the catalytic subunits, CAPN1 and CAPN2, which form calpain-1 and calpain-2 isozymes, respectively, together with their common regulatory subunit, CAPNS1 (28-30). The substrate selectivity of those conventional calpains is currently unclear, but they exhibit enzymatically distinct properties. Indeed, calpain- 1 and calpain-2 require micromolar and millimolar levels of $\mathrm{Ca}^{2+}$ for half-maximal activation, respectively. It was reported that a variety of biophysical and biochemical stimuli elevate intracellular $\mathrm{Ca}^{2+}$ levels (31-33); therefore, the activity of these protease systems has been the focus of numerous pathophysiologic studies $(13,14)$. Our previous investigation indicated that oxidized or enzymatically modified LDL or its component, lysophosphatidylcholine, induced calpain-2; accordingly, this molecule is enriched in ECs in human and mouse atheromas (34). Calpain-2 exerts pro-atherogenic roles because the calpain inhibitors calpeptin and ALLM counteracted aortic atherosclerotic development in high cholesterol diet-fed $\mathrm{Ldlr}^{-/-}$mice. It was also reported that the calpain inhibitor BDA-410 inhibited atherosclerotic development in angiotensin II-infused $\mathrm{Ldlr}^{-/-}$mice (35). Furthermore, angiotensin II- or hypercholesterolemia-induced atherosclerosis in $\mathrm{Ldlr}^{-/-}$mice was suppressed by the transgenic overexpression of calpastatin; in contrast, abdominal aneurysmal formation, a complication of angiotensin II-infused mice, was unaltered (36). A study using angiotensin II-infused mice showed that calpain-1 in myeloids and calpain-2 in leukocytes were responsible for atherogenesis (36). Collectively, conventional calpains participate in pathophysiologic regulation in ECs and leukocytes, thereby facilitating atherosclerosis.
Our previous data identified that unconventional calpains also contribute to atherogenesis (37). Expression analysis in $\mathrm{Ldlr}^{-/-}$ mice showed that Capn2, Capn6, and Capn9 were upregulated in atheroprone aortae. Capn6 deficiency decelerated the progression of atherosclerosis in $\mathrm{Ldlr}^{-/-}$mice, while Capn 9 deficiency did not have any effect. Calpain- 6 is a non-proteolytic calpain because the cysteine residue in its $\mathrm{CysPc}$ domain is substituted with a lysine (28-30). This molecule is expressed preferentially in macrophages in advanced atheromas in humans and mice (37). Interestingly, calpain- 6 was originally identified in the skeletal muscle, cartilage, and heart of a murine fetus (38); however, its expression is absent in adult tissues with the exception of the placenta under non-disease conditions (39). Therefore, calpain- 6 might be potentiated under developmental and pathogenic conditions. Regarding atherosclerosis, it is likely that calpain-6 is upregulated in macrophages after cells infiltrate into lesions, whereas it is absent in bone marrow cells (37). Furthermore, calpain-6 induction can be reproduced in cultured bone marrow-derived macrophages by cytokine stimulation. In particular, tumor necrosis factor- $\alpha$ markedly induced calpain-6. Atherogenesis appears to be dependent upon myeloid Capn6 but not upon other cell lineages in mice. Therefore, the proatherogenic transformation of macrophages by calpain- 6 is responsible for atherosclerotic disease.

\section{CONTRIBUTION OF DEFECTIVE PROTEIN CATABOLISM TO ATHEROGENIC INFLAMMATION}

During the development of atherosclerosis, the dysfunction of ECs and fibrogenicity of VSMCs, as well as cholesterol deposition and proinflammatory responses in macrophages are driven by defective protein catabolism (Figure 1; Table 1). The cell type-specific contribution of these defects to atherosclerosis is discussed below.

\section{Vascular ECs}

Endothelial cells cover the luminal surface of blood vessels and act as a physical barrier to limit the infiltration of active plasma factors through the vascular wall. It is well known that the endothelial barrier is disorganized in atheroprone arteries because of inflammatory insults (1), which accelerate the recruitment of immune cells that subsequently promote proinflammatory responses in the vessels. It is noteworthy that immune cells can also infiltrate atheroprone vascular walls through the vasa vasorum (40). Previous studies identified three major types of interendothelial junctions: tight junctions, gap junctions, and adherence junctions (41). In human umbilical vein ECs, tight junctions are present in up to $20 \%$ of the total junctional complexes, while adherence junctions account for approximately 90\% (42). Vascular endothelial (VE)-cadherin forms adherence junctions in a $\mathrm{Ca}^{2+}$-dependent homophilic manner (43). Furthermore, vascular permeability in mice was elevated by the administration of anti-VE-cadherin neutralizing antibody, whereas this treatment had no effect on other types of junctions in ECs (44); thus, adherence junctions have a pivotal role in barrier maintenance in ECs. Our previous investigations identified that administration of the calpain 


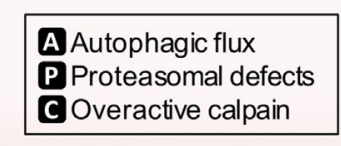

Lumen

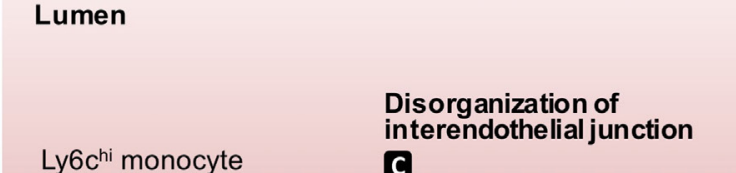

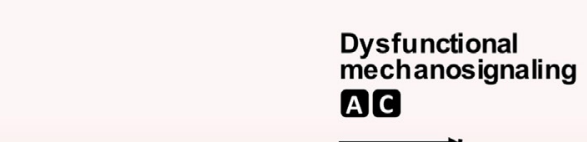

mechanosignaling AC

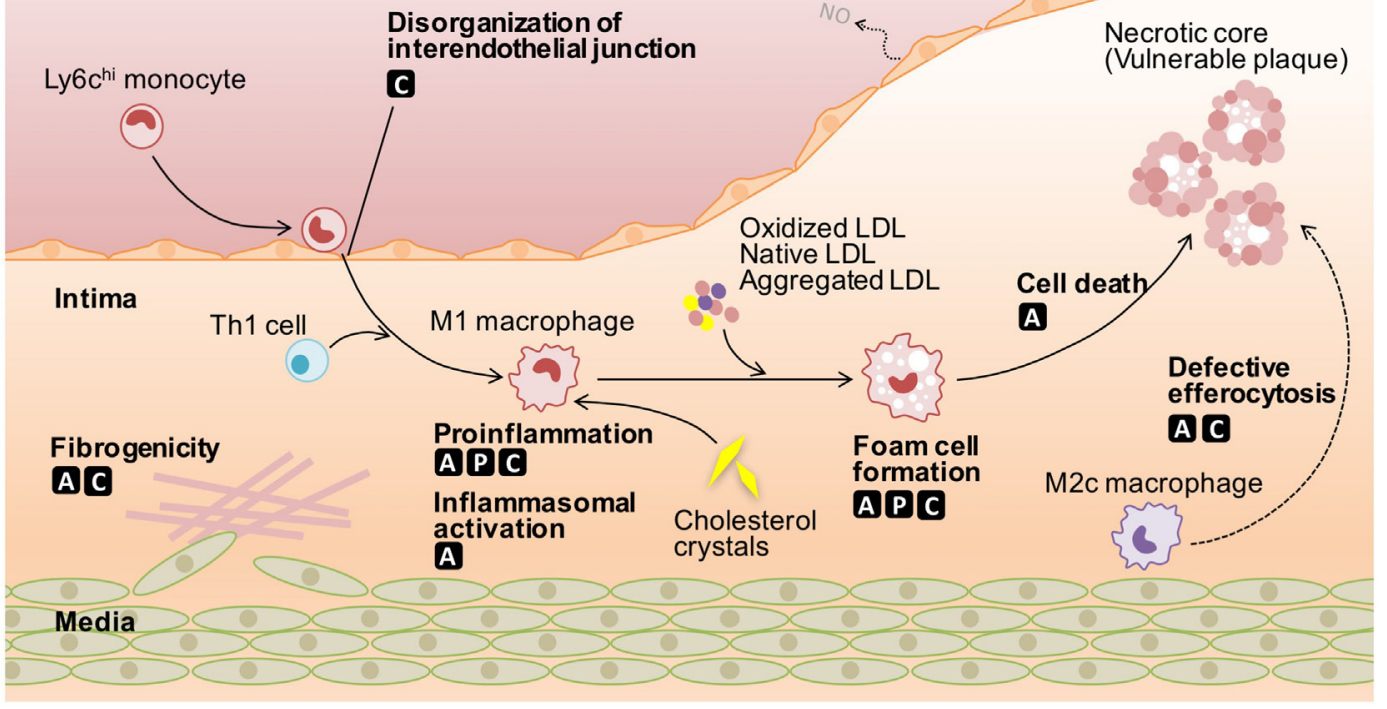

FIGURE 1 | Roles of defective protein catabolism in the pathogenesis of atherosclerosis. Autophagic flux leads to insufficient mechanosignaling in endothelial cells (ECs), impaired phenotypic conversion in vascular smooth muscle cells (VSMCs), proinflammatory actions in macrophages, and defective efferocytosis, thereby accelerating the onset of atherosclerosis. Proteasomal defects induce the depletion of nitric oxide in ECs and harmful proinflammatory responses in macrophages, despite protecting cells from apoptotic and non-apoptotic cell death. Overactivation of conventional calpains leads to impaired EC integrity, VSMC phenotypic conversion, and increased macrophage atherogenicity. Calpain-6 contributes to efferocytic pathways in macrophages. LDL, low-density lipoprotein; NO, nitric oxide.

inhibitors calpeptin and $N$-acetyl-Leu-Leu-methional (calpain inhibitor II) recovered the dysfunction of the EC barrier in dyslipidemic mice (34). Mechanistically, a juxtamembrane domain of VE-cadherin is proteolyzed by calpain-2, thereby dissociating $\beta$-catenin from the VE-cadherin complex, leading to the destabilization of adherence junctions. As a result, macrophage infiltration and the subsequent development of atherosclerosis in the dyslipidemic mice were suppressed by pharmacological inhibition of conventional calpains. Calpain proteolyzes E-cadherin and $\mathrm{N}$-cadherin; thus, calpain systems regulate the posttranslational processing of cadherin family proteins $(45,46)$. In contrast to the calpain family, the contribution of autophagy and proteasomal systems to atherosclerotic endothelial barrier dysfunctions has not been reported to date, although these pathways were reported to participate in the disorganization of tight junctions in septic pulmonary ECs (47) and ischemic cerebral ECs (48).

Endothelial nitric oxide (NO) production is crucial for ensuring vascular integrity. NO reacts with superoxide to neutralize its toxicity; therefore, a reduction of $\mathrm{NO}$ bioavailability facilitates ROS-dependent vascular inflammation. Furthermore, uncoupled endothelial nitric oxide synthase (eNOS), which generates superoxide radicals instead of NO, is largely responsible for ROS production even in atheroprone vessels $(49,50)$, in addition to xanthine oxidase and NADPH oxidase. It was reported that conventional calpains were responsible for physiological NO production in ECs. Indeed, calpain inhibitors $\mathrm{N}$-acetyl-leucylleucyl-norleucinal (calpain inhibitor I) or calpeptin prevented vascular endothelial growth factor (VEGF)-driven NO production in cultured ECs (51). Furthermore, VEGF accelerated the translocation of calpain to the plasma membrane to form an ezrin-containing molecular complex. Accordingly, AKT, AMPdependent kinase (AMPK), and eNOSs1179 were phosphorylated through the molecular complex, which is indispensable for NO production in ECs. In addition to VEGF-driven NO synthesis, disorders of NO production under pathophysiologic conditions might be caused by the dysregulation of calpain systems. Yu et al. documented that the administration of calpain inhibitor I increased the activity and protein expression of aortic eNOS in high fat diet-fed rats along with the elevation of NO (52) indicating excessive calpain activity may disturb NO synthesis in ECs. Herrmann et al. reported that in contrast to calpain inhibitors, the chronic administration of the proteasome inhibitor MLN-273 increased oxidative stress and exacerbated the development of atherosclerosis in pigs fed a high cholesterol 
TABLE 1 | Protein catabolism defects and atherosclerosis-related events.

\begin{tabular}{|c|c|c|c|c|}
\hline & Cell type & Events & Related processes & Reference \\
\hline \multirow[t]{5}{*}{ Autophagic flux } & ECs & NO depletion & Mechanotransduction & $(56)$ \\
\hline & & Endothelial NO synthase uncoupling & Mechanotransduction & $(57)$ \\
\hline & VSMCs & Fibrogenic response & VSMC transformation to synthetic phenotype & $(64)$ \\
\hline & Macrophages & Vascular inflammation & Inflammasome secretion of IL-1 $\beta$ & $(17)$ \\
\hline & & Impaired efferocytosis & Efferocytic clearance of dead cells & (18) \\
\hline \multirow[t]{3}{*}{ Proteasomal defects } & ECs & Impaired vasodilation & NO production & $(25)$ \\
\hline & Macrophages & Proinflammation & NF-кB activation (inhibitor $\kappa \mathrm{B}$ degradation) & $(76)$ \\
\hline & & Vascular protection? & Anti-apoptosis & $(10)$ \\
\hline \multirow[t]{8}{*}{ Overactive calpain } & ECs & Barrier dysfunction & VE-cadherin disorganization & (34) \\
\hline & & Endothelial disintegrity & NO depletion & (52) \\
\hline & VSMCs & Arterial fibrosis & Upregulation of $\mathrm{NF}-\kappa \mathrm{B}$ signals & $(65)$ \\
\hline & & Age-associated vascular fibrosis & VSMC transformation to synthetic phenotype & $(66)$ \\
\hline & & Vascular calcification & VSMC transformation to synthetic phenotype & $(67)$ \\
\hline & & Carotid restenosis & VSMC transformation to synthetic phenotype & (68) \\
\hline & Macrophages & Vascular inflammation & Upregulation of NF- $\mathrm{kB}$ signals & $(36)$ \\
\hline & & Impaired efferocytosis & mRNA splicing defects (calpain-6) & (37) \\
\hline
\end{tabular}

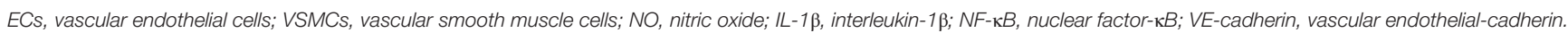

diet (25). In vitro experiments further showed that inhibition of the proteasome prevented EC-dependent vasodilation in isolated coronary arteries. Collectively, protein catabolism pathways are critical for endothelial NO production. It appears that proteasomal systems ensure NO synthesis, while calpain systems act as a negative regulator for NO production in atheroprone vessels.

It is widely known that fluid shear stress, the blood flowgenerated dragging force tangential to the EC surface, is a critical determinant for atherosclerosis susceptibility in blood vessels. This is because shear stress-dependent pathways in ECs are involved in barrier regulation and NO production (53). Interestingly, aortic arch, coronary artery, and aortic bifurcation, where blood flow is disturbed, are proatherogenic; thus, atherosclerosis susceptibility in blood vessels is largely dependent upon the vascular architecture because of the limited EC mechanotransduction in these regions (54). Yang et al. reported that physiological fluid shear stress sustained substantial autophagic activity in cultured ECs, but was reduced in cells subjected to low shear stress (55). Shear stress-induced eNOS phosphorylation and NO production were markedly blunted by autophagic deficiency (56). Similar to impaired NO production, the loss of autophagic activity potentiates ROS and cytokine production in ECs, suggesting that autophagy may play a suppressive role in eNOS uncoupling. Consistent with these results, autophagic inhibition by 3-methyladenine downregulated eNOS expression in sheared carotid arteries in rabbit, while autophagic activation by rapamycin potentiated eNOS expression (57). In addition to shear stress-dependent autophagic regulation, we previously showed that endothelial calpain-2 was activated in response to physiologic shear stress in cultured ECs (58). This activity was mediated through $\mathrm{Ca}^{2+}$ influx and phosphatidylinositol 3-kinase and was responsible for EC alignment in the direction of the blood flow. Interestingly, calpain-2 is enriched in specific vascular regions, such as the lesser curvature of the aortic arch and origin of vascular branch, in normal physiological murine aortae, where the blood flow is structurally disturbed (59). Thus, the autophagic and calpain systems orchestrate endothelial mechanotransduction.

\section{Vascular Smooth Muscle Cells}

It was reported that cardiovascular defects such as hypertension and atherosclerosis are strongly related to age-associated alterations in arterial structure and function $(60,61)$. The hallmark of such disorders is the stiffening of large arteries caused by ECM remodeling and vascular calcification and is triggered by the phenotypic conversion of contractile VSMCs to a synthetic phenotype. Mechanistically, synthetic VSMCs orchestrate elastin fragmentation as well as the synthesis and degradation of collagen through ROS-dependent inflammatory cascades. It was reported that RhoA, TGF- $\beta$, Notch, and integrin-matrix pathways modulated SMC differentiation (62) and that platelet-derived growth factor (PDGF) orchestrated the conversion of VSMCs to a contractile state along with autophagic activation (63). The pharmacological intervention of autophagy appears to inhibit the PDGF-induced phenotypic conversion in VSMCs, indicating the contribution of autophagic signals to the conversion. Interestingly, VSMC-specific deficiency of autophagy exacerbated high fat diet-induced atherosclerosis in $A p o e^{-/-}$mice, which was accompanied by a thickening of the fibrous cap (64). Furthermore, the autophagic flux resulted in an increase in collagen content, TGF- $\beta$ expression, and matrix metalloproteinase-9 in VSMCs. Thus, autophagy in VSMCs is protective in atherogenesis, but augments plaque vulnerability.

In addition to autophagy, calpain systems were reportedly associated with the phenotypic regulation in VSMCs. The transgenic overexpression of calpastatin antagonized arterial fibrosis and hypertrophy in mice infused with angiotensin II (65) by reduced MMP levels and the downregulation of NF- $\mathrm{KB}$ signals in arterial media. Similarly, the age-associated overactivation of calpain-1 in VSMCs participated in the upregulation of MMP2 and VSMC motility (66). Furthermore, osteopontin and 
osteonectin levels in VSMCs were reduced by the overexpression of calpain-1 in rats, inducing the calcification and fibrosis in the arteries of aged rats (67). Furthermore, VSMC proliferation and collagen synthesis were accelerated during ligation-induced carotid restenosis in mice, which was opposed by the transgenic overexpression of calpastatin (68). Therefore, calpain systems have a pivotal role in inflammation-related fibrogenic responses in VSMCs.

\section{Macrophages}

Macrophages exert an essential role in the pathogenesis and progression of atherosclerosis (69). Bone marrow-derived circulating monocytes can be divided into two subsets, LY6C ${ }^{\text {hi }}$ and LY6C ${ }^{\text {low }}$, in mice (70-72). The increased number of circulating monocytes in hypercholesterolemic mice is responsible for the expansion of the LY6C ${ }^{\text {hi }}$ subset (70), which is a major source of recruited macrophages in atheromas. In the early stage of atherosclerosis, circulating monocytes adhere to luminal ECs and migrate into the intimal layer according to the gradient of cytokines/chemokines (1). Subsequently, monocytes are differentiated into classically (M1) and alternatively (M2) activated macrophage subsets. M1 subset is mainly associated with inflammatory responses in atherosclerotic lesions and is derived from LY6C ${ }^{\text {hi }}$ monocytes (70-72). The M2 subset can be further divided into at least 4 subgroups, M2a, M2b, M2c, and M2d, which participate in tissue repair and inflammatory resolution in some instances (73). Foam cells in atherosclerotic lesions are mostly derived from the M1 subset, which is mediated through receptordependent and receptor-independent uptake of LDL cholesterol. In contrast, M2c macrophages incorporate cholesterol by engulfing dead cells efferocytically (74). The balance of M1 and M2 polarization can be disrupted in the presence of inflammatory burden in atherosclerotic lesions at least in part by disturbed poly (ADP-ribose) polymerase- or Dll4/Notch-related signals (73). Because cytosolic-free cholesterol exhibits robust cytotoxicity, the excessive incorporation of cholesterol results in cell death in macrophages. Accordingly, the acceleration of cholesterol uptake in macrophages in atheroprone vascular walls increases the number of dying macrophages, thereby forming cholesterolenriched vulnerable atherosclerotic lesions (75). In addition to their defective cholesterol handling, atheroprone macrophages play central roles in innate immunity, including toll-like receptor and NOD-like receptor-mediated inflammasome signaling, in lesions (69). Although the protein catabolism pathway critically influences both cholesterol handling and inflammatory cascades in cells, cholesterol handling is not discussed in this issue. Please refer to our previous review articles for information on proatherogenic cholesterol handling in macrophages by defective cholesterol catabolism (12).

It was reported that macrophage-specific deficiency of autophagy by the deletion of Atg5 facilitated atherogenesis in

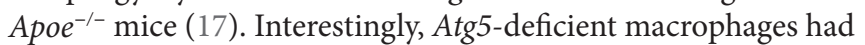
increased oxidative stress and enhanced inflammasome secretion of IL-1 $\beta$. The inflammasome systems in Atg5-null macrophages were potentiated by the addition of cholesterol crystals to the culture systems. It is likely that the inflammasome secretion of IL-1 $\beta$ by Atg5-null macrophages in atheromas may be potentiated by cholesterol crystals, because they are enriched in atherosclerotic lesions in Atg5-deficient mice. Furthermore, necrotic cores and oxidative stress in lesions of $\mathrm{Ldlr}^{-/-}$mice were enhanced by the macrophage-specific deletion of Atg5 (18). Autophagic activity in isolated macrophages was potentiated by proatherogenic stressors, such as 7-ketocholesterol or 1-(palmitoyl)-2-(5-keto-6-octenedioyl) phosphatidylcholine. Such autophagic activity suppressed apoptotic cell death by the reduction of endoplasmic reticulum stress and oxidative stress by NADPH oxidase 2. Furthermore, atherogenicity in macrophages was also mediated by proteasomal defects. For example, Brand et al. reported that the pharmacological inhibition of proteasomes prevented the proteolytic degradation of inhibitor $\kappa \mathrm{B} \alpha$, an endogenous inhibitor of NF- $\kappa \mathrm{B}$ transcriptional systems, by oxidized LDL in monocytes (76). This indicates that proteasomal inhibitors possess anti-inflammatory effects in atherosclerosis. In contrast, it was reported that oxidized LDL-stimulated macrophages showed increased ubiquitination activity, which suppressed apoptosis (10). Similarly, the complex mode of cell death in VSMCs was abrogated by 7-ketocholesterolindced proteasomal activity (11). Thus, proteasomal activity may be involved in cell survival under stressed conditions, while it participates in cytotoxic inflammatory burden. In addition to the autophagic and proteasomal flux, the participation of calpain systems in inflammatory cascades of macrophages was proposed. It appears that calpain can proteolytically degrade inhibitor $\kappa \mathrm{B} \alpha$, thereby accelerating NF- $\kappa \mathrm{B}$ signaling (77); thus, the overactivation of calpain systems potentiates inflammatory responses. The transduction of CAST prevented the production of inflammatory cytokines in peritoneal macrophages isolated from high fat diet-fed Apoe ${ }^{-/-}$mice (36). Collectively, macrophages can be susceptible to proatherogenic burden by the overactivation of calpain systems. Whether defective protein catabolism influences macrophage polarity is currently unclear and, therefore, should be studied in the future.

Vulnerable atherosclerotic lesions harbor vast number of inflammatory cells, a necrotic core, and a thin fibrous cap (78). In particular, expansion of the cholesterol-enriched necrotic core by the accumulation of dead cells weakens the physical stability of plaques. It was reported that macrophages engulf and remove cellular corpses in lesions through efferocytic activity, thereby reducing the necrotic core (78). Furthermore, the efferocytic actions of macrophages participate in the reprogramming of cells toward anti-inflammatory phenotypes, leading to the resolution of inflammation. Importantly, the clearance of dead cells by efferocytosis is limited in advanced atheromas, because "eat-me/ find-me" signals in apoptotic cells and phagocytosis pathways in macrophages are prevented in the lesions (78). Thus, the coordination of integrated cell death signals in dying cells and phagocytic signals in phagocytes are necessary for efferocytosis. In the case of mammary gland involution in pregnancy, efferocytic activity was accelerated in mammary epithelial cells, resulting in remodeling of the tissues and the production of milk (79). Mammary epithelial-specific Atg7 deficiency markedly reduced efferocytic activity, Rac1 activity, and MertK expression; thus, autophagic signaling is associated with efferocytosis-associated tissue remodeling. In the case of atherosclerosis, autophagic defects caused by Atg5 deficiency reduced the efficiency of the 
efferocytic clearance of dead cells, leading to an expansion of the necrotic core (18). It is likely that Atg5 deficiency in dying cells, but not phagocytic macrophages, prevented the efficiency of efferocytosis. Therefore, the autophagic disorder in dying cells or phagocytic macrophages induced defects in efferocytosis, although this needs to be elucidated in the future. While the participation of conventional calpains in efferocytosis has not been reported to date, calpain- 6 seems to be involved in this event. Indeed, the efferocytic clearance of dead cells by cultured macrophages was modestly inhibited by Capn6 deficiency (37). Accordingly, the deletion of Capn6 resulted in a reduction of necrotic cores in atheromas in $\mathrm{Ldlr}^{-/-}$mice, which was accompanied by the upregulation of Racl protein in the cells. Racl prevention by a pharmacological inhibitor suppressed efferocytic activity in macrophages (80); therefore, it is interpreted that efferocytic activity in lesional macrophages was prevented by calpain-6, probably through Rac1 downregulation. Because conventional calpains are associated with cellular dynamic processes, such as migration (81) and trafficking of intracellular vesicles (82), it will be interesting to investigate the participation of these calpains in efferocytosis.

\section{TARGETING DEFECTIVE PROTEIN CATABOLISM IN ATHEROSCLEROSIS}

As stated above, autophagic disorders cause insufficient mechanosignaling in ECs, impaired phenotypic conversion in VSMCs, inflammatory action in macrophages, and defective efferocytosis, thereby increasing the susceptibility to atherosclerosis (Figure 1). Therefore, recovering autophagic activity may be a promising treatment for the atherosclerosis burden. One possible candidate for atherosclerosis intervention is mTOR, a robust autophagic suppressor. mTOR inhibitors normally exhibit proautophagic activity thereby suppressing atherosclerosis (83). For example, atherosclerosis in rabbits fed a high cholesterol diet (84), or in Apoe $^{-/-}$(85) and $\mathrm{Ldlr}^{-/-}$mice (86) was markedly inhibited by oral administration of the mTOR inhibitor rapamycin. Clinical trials of several mTOR inhibitors for cancer treatment are currently underway (87). Based on our current knowledge, it is expected that the use of mTOR inhibitors will also be beneficial for treating cardiovascular disorders.

Proteasomal activation induces harmful proinflammatory responses in macrophages but protects cells from apoptotic and nonapoptotic cell death, whereas proteasomal inhibition leads to the depletion of EC-derived NO (Figure 1). Thus, based on animal experiments, it is likely that inhibiting proteasomes will have a dual action. Accordingly, the clinical applications of proteasomal inhibitors for treating atherosclerosis are currently unclear, even though atherosclerotic diseases can be reversed by moderate levels of proteasomal inhibition (27). In addition to these inhibitors, endogenous activators of proteasomes, PA28, PA200, and PA700, as well as chemical proteasome activators, are currently available (88). The beneficial effects of PA28 overexpression in a neuronal cell model of Huntington's disease were reported (89). Restoring proteasomal activity by activators should be investigated in future atherosclerosis studies.
Both calpain-6 and conventional calpains are involved in atheroprone events in lesional macrophages. Whereas conventional calpains lead to impaired EC integrity, phenotypic conversion of VSMCs and increased macrophage atherogenicity, calpain- 6 contributes to cholesterol uptake and efferocytic processes in phagocytic macrophages (Table 1). Importantly, the majority of proatherogenic events can be prevented by loss-offunction calpains $(12,30)$, indicating that targeting calpain systems is a promising approach for atherosclerosis therapy. Clinical trials of candidate compounds for the prevention of conventional calpains are currently underway, especially for neurodegenerative diseases (30). Based on our knowledge from basic research, the repositioning of these agents to the cardiovascular field may be highly beneficial. Isozyme selectivity of available calpain inhibitors, in particular, those regarding unconventional isozymes, are mostly unclear. Furthermore, it is expected that available inhibitors will not block calpain- 6 because this molecule exhibits physiologic/pathophysiologic functions through its capacity to bind to other functional molecules, but not through its proteolytic activity. Evaluation of the isozyme selectivity of available inhibitors among unconventional calpains and the development of subtype selective inhibitors will be indispensable for calpaintargeted therapy in the future.

In conclusion, respective protein catabolism pathways, autophagy, proteasomes, and calpain systems, have unique properties under proatherogenic conditions. It appears that autophagy and proteasomes have fundamental roles in cell survival, while calpains appear to be dispensable. In contrast, autophagy and proteasomal systems are limited during the progression of atherosclerosis, while proatherogenic stressors activate calpains in some instances. It is noteworthy that apoptotic cell death was accelerated by the calpain-mediated proteolysis of Atg5 (90). Therefore, exploring the interrelations among the catabolism pathways in atherosclerosis is required for a better understanding of the pathogenic roles of these protein catabolism pathways.

\section{AUTHOR CONTRIBUTIONS}

TM conceived, designed, appraised the literature, and wrote the manuscript. AM reviewed and revised the manuscript.

\section{ACKNOWLEDGMENTS}

We thank J. Ludovic Croxford, Ph.D., from Edanz Group (http:// www.edanzediting.com/ac) for editing a draft of this manuscript.

\section{FUNDING}

This study was supported in part by Japan Society for the Promotion of Science KAKENHI Grant Number 17K09869 (to AM), Japan Society for the Promotion of Science KAKENHI Grant Number 15K09418 (to TM), and research grants from Takeda Science Foundation, Banyu Life Science Foundation International, NOVARTIS Foundation, Ono Medical Research Foundation, Japan Heart Foundation \& Astellas Grant for Research on Atherosclerosis Update, and SENSHIN Medical Research Foundation (all to TM). 


\section{REFERENCES}

1. Ross R. Atherosclerosis - an inflammatory disease. N Engl J Med (1999) 340:115-26. doi:10.1056/NEJM199901143400207

2. Weber C, Noels H. Atherosclerosis: current pathogenesis and therapeutic options. Nat Med (2011) 17:1410-22. doi:10.1038/nm.2538

3. Libby P, Aikawa M. Stabilization of atherosclerotic plaques: new mechanisms and clinical targets. Nat Med (2002) 8:1257-62. doi:10.1038/ nm1102-1257

4. Steinhubl SR. Why have antioxidants failed in clinical trials? Am J Cardiol (2008) 101:14D-9D. doi:10.1016/j.amjcard.2008.02.003

5. Kikuchi K, Tanaka E, Murai Y, Tancharoen S. Clinical trials in acute ischemic stroke. CNS Drugs (2014) 28:929-38. doi:10.1007/s40263014-0199-6

6. Mizushima N, Komatsu M. Autophagy: renovation of cells and tissues. Cell (2011) 147:728-41. doi:10.1016/j.cell.2011.10.026

7. Lavandero S, Chiong M, Rothermel BA, Hill JA. Autophagy in cardiovascular biology. J Clin Invest (2015) 125:55-64. doi:10.1172/JCI73943

8. Tanaka K, Matsuda N. Proteostasis and neurodegeneration: the roles of proteasomal degradation and autophagy. Biochim Biophys Acta (2014) 1843:197-204. doi:10.1016/j.bbamcr.2013.03.012

9. Qureshi N, Vogel SN, Van Way C III, Papasian CJ, Qureshi AA, Morrison DC. The proteasome: a central regulator of inflammation and macrophage function. Immunol Res (2005) 31:243-60. doi:10.1385/IR:31:3:243

10. Vieira O, Escargueil-Blanc I, Jürgens G, Borner C, Almeida L, Salvayre R, et al. Oxidized LDLs alter the activity of the ubiquitin-proteasome pathway: potential role in oxidized LDL-induced apoptosis. FASEB J (2000) 14: 532-42. doi:10.1096/fj.1530-6860

11. Martinet W, De Bie M, Schrijvers DM, De Meyer GR, Herman AG, Kockx MM. 7-Ketocholesterol induces protein ubiquitination, myelin figure formation, and light chain 3 processing in vascular smooth muscle cells. Arterioscler Thromb Vasc Biol (2004) 24:2296-301. doi:10.1161/01.ATV.0000146266.65820.a1

12. Miyazaki T, Miyazaki A. Emerging roles of calpain proteolytic systems in macrophage cholesterol handling. Cell Mol Life Sci (2017) 74:3011-21. doi:10.1007/s00018-017-2528-7

13. Miyazaki T, Miyazaki A. Dysregulation of calpain proteolytic systems underlies degenerative vascular disorders. J Atheroscler Thromb (2017). doi:10.5551/ jat.RV17008

14. Miyazaki T, Koya T, Kigawa Y, Oguchi T, Lei XF, Kim-Kaneyama JR, et al. Calpain and atherosclerosis. J Atheroscler Thromb (2013) 20:228-37. doi: $10.5551 /$ jat. 14787

15. Levine B, Kroemer G. Autophagy in the pathogenesis of disease. Cell (2008) 132:27-42. doi:10.1016/j.cell.2007.12.018

16. Maiuri MC, Zalckvar E, Kimchi A, Kroemer G. Self-eating and self-killing: crosstalk between autophagy and apoptosis. Nat Rev Mol Cell Biol (2007) 8:741-52. doi:10.1038/nrm2239

17. Razani B, Feng C, Coleman T, Emanuel R, Wen H, Hwang S, et al. Autophagy links inflammasomes to atherosclerotic progression. Cell Metab (2012) 15:534-44. doi:10.1016/j.cmet.2012.02.011

18. Liao X, Sluimer JC, Wang Y, Subramanian M, Brown K, Pattison JS, et al. Macrophage autophagy plays a protective role in advanced atherosclerosis. Cell Metab (2012) 15:545-53. doi:10.1016/j.cmet.2012.01.022

19. Sijts EJ, Kloetzel PM. The role of the proteasome in the generation of MHC class I ligands and immune responses. Cell Mol Life Sci (2011) 68:1491-502. doi:10.1007/s00018-011-0657-y

20. DeSalle LM, Pagano M. Regulation of the G1 to $S$ transition by the ubiquitin pathway. FEBS Lett (2001) 490:179-89. doi:10.1016/S0014-5793(01)02121-4

21. Kimura Y, Tanaka K. Regulatory mechanisms involved in the control of ubiquitin homeostasis. J Biochem (2010) 147:793-8. doi:10.1093/jb/mvq044

22. Zheng Q, Huang T, Zhang L, Zhou Y, Luo H, Xu H, et al. Dysregulation of ubiquitin-proteasome system in neurodegenerative diseases. Front Aging Neurosci (2016) 8:303. doi:10.3389/fnagi.2016.00303

23. Herrmann J, Lerman LO, Lerman A. On to the road to degradation: atherosclerosis and the proteasome. Cardiovasc Res (2010) 85:291-302. doi:10.1093/ cvr/cvp333

24. Herrmann J, Soares SM, Lerman LO, Lerman A. Potential role of the ubiquitin-proteasome system in atherosclerosis: aspects of a protein quality disease. J Am Coll Cardiol (2008) 51:2003-10. doi:10.1016/j.jacc.2008.02.047
25. Herrmann J, Saguner AM, Versari D, Peterson TE, Chade A, Olson M, et al. Chronic proteasome inhibition contributes to coronary atherosclerosis. Circ Res (2007) 101:865-74. doi:10.1161/CIRCRESAHA.107.152959

26. Versari D, Herrmann J, Gössl M, Mannheim D, Sattler K, Meyer FB, et al. Dysregulation of the ubiquitin-proteasome system in human carotid atherosclerosis. Arterioscler Thromb Vasc Biol (2006) 26:2132-9. doi:10.1161/ 01.ATV.0000232501.08576.73

27. Wilck N, Fechner M, Dreger H, Hewing B, Arias A, Meiners S, et al. Attenuation of early atherogenesis in low-density lipoprotein receptor-deficient mice by proteasome inhibition. Arterioscler Thromb Vasc Biol (2012) 32:1418-26. doi:10.1161/ATVBAHA.112.249342

28. Goll DE, Thompson VF, Li H, Wei W, Cong J. The calpain system. Physiol Rev (2003) 83:731-801. doi:10.1152/physrev.00029.2002

29. Ono Y, Sorimachi H. Calpains: an elaborate proteolytic system. Biochim Biophys Acta (2012) 1824:224-36. doi:10.1016/j.bbapap.2011.08.005

30. Ono Y, Saido TC, Sorimachi H. Calpain research for drug discovery: challenges and potential. Nat Rev Drug Discov (2016) 15:854-76. doi:10.1016/ j.bbapap.2011.08.005

31. De Bock M, Wang N, Decrock E, Bol M, Gadicherla AK, Culot M, et al. Endothelial calcium dynamics, connexin channels and blood-brain barrier function. Prog Neurobiol (2013) 108:1-20. doi:10.1016/j.pneurobio. 2013.06.001

32. Miyazaki T, Ohata H, Yamamoto M, Momose K. Spontaneous and flowinduced $\mathrm{Ca}^{2+}$ transients in retracted regions in endothelial cells. Biochem Biophys Res Commun (2001) 281:172-9. doi:10.1006/bbrc.2001.4331

33. Miyazaki T, Honda K, Ohata $\mathrm{H}$. Modulation of $\mathrm{Ca}^{2+}$ transients in cultured endothelial cells in response to fluid flow through alphaV integrin. Life Sci (2007) 81:1421-30. doi:10.1016/j.lfs.2007.08.041

34. Miyazaki T, Taketomi Y, Takimoto M, Lei XF, Arita S, Kim-Kaneyama JR, et al. $\mathrm{m}$-Calpain induction in vascular endothelial cells on human and mouse atheromas and its roles in VE-cadherin disorganization and atherosclerosis. Circulation (2011) 124:2522-32. doi:10.1161/CIRCULATIONAHA. 111.021675

35. Subramanian V, Uchida HA, Ijaz T, Moorleghen JJ, Howatt DA, Balakrishnan A. Calpain inhibition attenuates angiotensin II-induced abdominal aortic aneurysms and atherosclerosis in low-density lipoprotein receptor-deficient mice. J Cardiovasc Pharmacol (2012) 59:66-76. doi:10.1097/FJC.0b013e318235d5ea

36. Howatt DA, Balakrishnan A, Moorleghen JJ, Muniappan L, Rateri DL, Uchida HA, et al. Leukocyte calpain deficiency reduces angiotensin II-induced inflammation and atherosclerosis but not abdominal aortic aneurysms in mice. Arterioscler Thromb Vasc Biol (2016) 36:835-45. doi:10.1161/ ATVBAHA.116.307285

37. Miyazaki T, Tonami K, Hata S, Aiuchi T, Ohnishi K, Lei XF, et al. Calpain-6 confers atherogenicity to macrophages by dysregulating pre-mRNA splicing. J Clin Invest (2016) 126:3417-32. doi:10.1172/JCI85880

38. Tonami K, Hata S, Ojima K, Ono Y, Kurihara Y, Amano T, et al. Calpain-6 deficiency promotes skeletal muscle development and regeneration. PLoS Genet (2013) 9:e1003668. doi:10.1371/journal.pgen.1003668

39. Dear N, Matena K, Vingron M, Boehm T. A new subfamily of vertebrate calpains lacking a calmodulin-like domain: implications for calpain regulation and evolution. Genomics (1997) 45:175-84. doi:10.1006/geno.1997.4870

40. Campbell KA, Lipinski MJ, Doran AC, Skaflen MD, Fuster V, McNamara CA. Lymphocytes and the adventitial immune response in atherosclerosis. Circ Res (2012) 110:889-900. doi:10.1161/CIRCRESAHA.111.263186

41. Mehta D, Malik AB. Signaling mechanisms regulating endothelial permeability. Physiol Rev (2006) 86:279-367. doi:10.1152/physrev.00012.2005

42. Wójciak-Stothard B, Potempa S, Eichholtz T, Ridley AJ. Rho and Rac but not Cdc42 regulate endothelial cell permeability. J Cell Sci (2001) 114:1343-55.

43. Gavard J. Breaking the VE-cadherin bonds. FEBS Lett (2009) 583:1-6. doi:10.1016/j.febslet.2008.11.032

44. Corada M, Mariotti M, Thurston G, Smith K, Kunkel R, Brockhaus M, et al. Vascular endothelial-cadherin is an important determinant of microvascular integrity in vivo. Proc Natl Acad Sci U S A (1999) 96:9815-20. doi:10.1073/ pnas.96.17.9815

45. Kudo-Sakamoto Y, Akazawa H, Ito K, Takano J, Yano M, Yabumoto C, et al. Calpain-dependent cleavage of $\mathrm{N}$-cadherin is involved in the progression of post-myocardial infarction remodeling. J Biol Chem (2014) 289:19408-19. doi:10.1074/jbc.M114.567206 
46. Ye Y, Tian H, Lange AR, Yearsley K, Robertson FM, Barsky SH. The genesis and unique properties of the lymphovascular tumor embolus are because of calpain-regulated proteolysis of E-cadherin. Oncogene (2013) 32:1702-13. doi:10.1038/onc.2012.180

47. Zhang D, Zhou J, Ye LC, Li J, Wu Z, Li Y, et al. Autophagy maintains the integrity of endothelial barrier in LPS-induced lung injury. J Cell Physiol (2017) 233:688-98. doi:10.1002/jcp.25928

48. Li H, Gao A, Feng D, Wang Y, Zhang L, Cui Y, et al. Evaluation of the protective potential of brain microvascular endothelial cell autophagy on blood-brain barrier integrity during experimental cerebral ischemia-reperfusion injury. Transl Stroke Res (2014) 5:618-26. doi:10.1007/ s12975-014-0354-x

49. Nowak WN, Deng J, Ruan XZ, Xu Q. Reactive oxygen species generation and atherosclerosis. Arterioscler Thromb Vasc Biol (2017) 37:e41-52. doi:10.1161/ ATVBAHA.117.309228

50. Rochette L, Lorin J, Zeller M, Guilland JC, Lorgis L, Cottin Y, et al. Nitric oxide synthase inhibition and oxidative stress in cardiovascular diseases: possible therapeutic targets? Pharmacol Ther (2013) 140:239-57. doi:10.1016/ j.pharmthera.2013.07.004

51. Youn JY, Wang T, Cai H. An ezrin/calpain/PI3K/AMPK/eNOSs1179 signaling cascade mediating VEGF-dependent endothelial nitric oxide production. Circ Res (2009) 104:50-9. doi:10.1161/CIRCRESAHA.108.178467

52. Yu L, Yin M, Yang X, Lu M, Tang F, Wang H. Calpain inhibitor attenuates atherosclerosis and inflammation in atherosclerotic rats through eNOS/NO/ NF-kB pathway. Can J Physiol Pharmacol (2017). doi:10.1139/cjpp-2016-0652

53. Davies PF. Flow-mediated endothelial mechanotransduction. Physiol Rev (1995) 75:519-60.

54. VanderLaan PA, Reardon CA, Getz GS. Site specificity of atherosclerosis: site-selective responses to atherosclerotic modulators. Arterioscler Thromb Vasc Biol (2004) 24:12-22. doi:10.1161/01.ATV.0000105054.43931.f0

55. Yang Q, Li X, Li R, Peng J, Wang Z, Jiang Z, et al. Low shear stress inhibited endothelial cell autophagy through TET2 downregulation. Ann Biomed Eng (2016) 44:2218-27. doi:10.1007/s10439-015-1491-4

56. Bharath LP, Mueller R, Li Y, Ruan T, Kunz D, Goodrich R, et al. Impairment of autophagy in endothelial cells prevents shear-stress-induced increases in nitric oxide bioavailability. Can J Physiol Pharmacol (2014) 92:605-12. doi:10.1139/cjpp-2014-0017

57. Guo F, Li X, Peng J, Tang Y, Yang Q, Liu L, et al. Autophagy regulates vascular endothelial cell eNOS and ET-1 expression induced by laminar shear stress in an ex vivo perfused system. Ann Biomed Eng (2014) 42:1978-88. doi:10.1007/ s10439-014-1033-5

58. Miyazaki T, Honda $\mathrm{K}$, Ohata $\mathrm{H}$. Requirement of $\mathrm{Ca}^{2+}$ influx- and phosphatidylinositol 3-kinase-mediated $\mathrm{m}$-calpain activity for shear stress-induced endothelial cell polarity. Am J Physiol Cell Physiol (2007) 293: C1216-25. doi:10.1152/ajpcell.00083.2007

59. Miyazaki T, Honda K, Ohata H. m-Calpain antagonizes RhoA overactivation and endothelial barrier dysfunction under disturbed shear conditions. Cardiovasc Res (2010) 85:530-41. doi:10.1093/cvr/cvp311

60. Lakatta EG. Arterial and cardiac aging: major shareholders in cardiovascular disease enterprises: part III: cellular and molecular clues to heart and arterial aging. Circulation (2003) 107:490-9. doi:10.1161/01.CIR.0000048894.99865.02

61. Nagai Y, Metter EJ, Earley CJ, Kemper MK, Becker LC, Lakatta EG, et al. Increased carotid artery intimal-medial thickness in asymptomatic older subjects with exercise-induced myocardial ischemia. Circulation (1998) 98:1504-9. doi:10.1161/01.CIR.98.15.1504

62. Mack CP. Signaling mechanisms that regulate smooth muscle cell differentiation. Arterioscler Thromb Vasc Biol (2011) 31:1495-505. doi:10.1161/ ATVBAHA.110.221135

63. Salabei JK, Cummins TD, Singh M, Jones SP, Bhatnagar A, Hill BG. PDGFmediated autophagy regulates vascular smooth muscle cell phenotype and resistance to oxidative stress. Biochem J (2013) 451:375-88. doi:10.1042/ BJ20121344

64. Grootaert MO, da Costa Martins PA, Bitsch N, Pintelon I, De Meyer GR, Martinet W, et al. Defective autophagy in vascular smooth muscle cells accelerates senescence and promotes neointima formation and atherogenesis. Autophagy (2015) 11:2014-32. doi:10.1080/15548627.2015.1096485

65. Letavernier E, Perez J, Bellocq A, Mesnard L, de Castro Keller A, Haymann JP, et al. Targeting the calpain/calpastatin system as a new strategy to prevent cardiovascular remodeling in angiotensin II-induced hypertension. Circ Res (2008) 102:720-8. doi:10.1161/CIRCRESAHA.107.160077

66. Jiang L, Wang M, Zhang J, Monticone RE, Telljohann R, Spinetti G, et al. Increased aortic calpain-1 activity mediates age-associated angiotensin II signaling of vascular smooth muscle cells. PLoS One (2008) 3:e2231. doi:10.1371/ journal.pone.0002231

67. Jiang L, Zhang J, Monticone RE, Telljohann R, Wu J, Wang M, et al. Calpain-1 regulation of matrix metalloproteinase 2 activity in vascular smooth muscle cells facilitates age-associated aortic wall calcification and fibrosis. Hypertension (2012) 60:1192-9. doi:10.1161/HYPERTENSIONAHA.112.196840

68. Tang L, Pei H, Yang Y, Wang X, Wang T, Gao E, et al. The inhibition of calpains ameliorates vascular restenosis through MMP2/TGF- $\beta 1$ pathway. Sci Rep (2016) 6:29975. doi:10.1038/srep29975

69. Moore KJ, Sheedy FJ, Fisher EA. Macrophages in atherosclerosis: a dynamic balance. Nat Rev Immunol (2013) 13:709-21. doi:10.1038/nri3520

70. Swirski FK, Libby P, Aikawa E, Alcaide P, Luscinskas FW, Weissleder R, et al. Ly- $6 \mathrm{C}^{\mathrm{hi}}$ monocytes dominate hypercholesterolemia-associated monocytosis and give rise to macrophages in atheromata. J Clin Invest (2007) 117:195-205. doi:10.1172/JCI29950

71. Tacke F, Alvarez D, Kaplan TJ, Jakubzick C, Spanbroek R, Llodra J, et al. Monocyte subsets differentially employ CCR2, CCR5, and CX3CR1 to accumulate within atherosclerotic plaques. J Clin Invest (2007) 117:185-94. doi:10.1172/JCI28549

72. Yvan-Charvet L, Pagler T, Gautier EL, Avagyan S, Siry RL, Han S, et al. ATPbinding cassette transporters and HDL suppress hematopoietic stem cell proliferation. Science (2010) 328:1689-93. doi:10.1126/science.1189731

73. Nakano T, Fukuda D, Koga J, Aikawa M. Delta-like ligand 4-Notch signaling in macrophage activation. Arterioscler Thromb Vasc Biol (2016) 36:2038-47. doi:10.1161/ATVBAHA.116.306926

74. Getz GS, Reardon CA. Atherogenic lipids and macrophage subsets. Curr Opin Lipidol (2015) 26:357-61. doi:10.1097/MOL.0000000000000217

75. Silvestre-Roig C, de Winther MP, Weber C, Daemen MJ, Lutgens E, Soehnlein O. Atherosclerotic plaque destabilization: mechanisms, models, and therapeutic strategies. Circ Res (2014) 114:214-26. doi:10.1161/ CIRCRESAHA.114.302355

76. Brand K, Eisele T, Kreusel U, Page M, Page S, Haas M, et al. Dysregulation of monocytic nuclear factor-kappa B by oxidized low-density lipoprotein. Arterioscler Thromb Vasc Biol (1997) 17:1901-9. doi:10.1161/01.ATV. 17.10.1901

77. Lin YC, Brown K, Siebenlist U. Activation of NF- $\mathrm{kB}$ requires proteolysis of

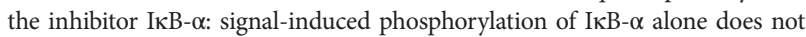
release active NF-kB. Proc Natl Acad Sci U S A (1995) 92:552-6. doi:10.1073/ pnas.92.2.552

78. Ortega-Gómez A, Perretti M, Soehnlein O. Resolution of inflammation: an integrated view. $E M B O \mathrm{Mol} \mathrm{Med} \mathrm{(2013)} \mathrm{5:661-74.} \mathrm{doi:10.1002/emmm.}$ 201202382

79. Teplova I, Lozy F, Price S, Singh S, Barnard N, Cardiff RD, et al. ATG proteins mediate efferocytosis and suppress inflammation in mammary involution. Autophagy (2013) 9:459-75. doi:10.4161/auto.23164

80. Tao H, Yancey PG, Babaev VR, Blakemore JL, Zhang Y, Ding L, et al. Macrophage SR-BI mediates efferocytosis via Src/PI3K/Racl signaling and reduces atherosclerotic lesion necrosis. JLipid Res (2015) 56:1449-60. doi:10.1194/jlr.M056689

81. Miyazaki T, Taketomi Y, Saito Y, Hosono T, Lei XF, Kim-Kaneyama JR, et al. Calpastatin counteracts pathological angiogenesis by inhibiting suppressor of cytokine signaling 3 degradation in vascular endothelial cells. Circ Res (2015) 116:1170-81. doi:10.1161/CIRCRESAHA.116.305363

82. Tsai JC, Lin YW, Huang CY, Lin CY, Tsai YT, Shih CM, et al. The role of calpain-myosin 9-Rab7b pathway in mediating the expression of toll-like receptor 4 in platelets: a novel mechanism involved in $\alpha$-granules trafficking. PLoS One (2014) 9:e85833. doi:10.1371/journal.pone.0085833

83. Martinet W, De Loof H, De Meyer GR. mTOR inhibition: a promising strategy for stabilization of atherosclerotic plaques. Atherosclerosis (2014) 233: 601-7. doi:10.1016/j.atherosclerosis.2014.01.040

84. Chen WQ, Zhong L, Zhang L, Ji XP, Zhang M, Zhao YX, et al. Oral rapamycin attenuates inflammation and enhances stability of atherosclerotic plaques in rabbits independent of serum lipid levels. Br J Pharmacol (2009) 156: 941-51. doi:10.1111/j.1476-5381.2008.00102.x 
85. Pakala R, Stabile E, Jang GJ, Clavijo L, Waksman R. Rapamycin attenuates atherosclerotic plaque progression in apolipoprotein $\mathrm{E}$ knockout mice: inhibitory effect on monocyte chemotaxis. J Cardiovasc Pharmacol (2005) 46:481-6. doi:10.1097/01.fjc.0000177985.14305.15

86. Zhao L, Ding T, Cyrus T, Cheng Y, Tian H, Ma M, et al. Low-dose oral sirolimus reduces atherogenesis, vascular inflammation and modulates plaque composition in mice lacking the LDL receptor. Br J Pharmacol (2009) 156:774-85. doi:10.1111/j.1476-5381.2008.00080.x

87. Xie J, Wang X, Proud CG. mTOR inhibitors in cancer therapy. F1000Res (2016) 5(F1000 Faculty Rev):2078. doi:10.12688/f1000research.9207.1

88. Huang L, Chen CH. Proteasome regulators: activators and inhibitors. Curr Med Chem (2009) 16:931-9. doi:10.2174/092986709787581860

89. Seo H, Sonntag KC, Kim W, Cattaneo E, Isacson O. Proteasome activator enhances survival of huntington's disease neuronal model cells. PLoS One (2007) 2:e238. doi:10.1371/journal.pone.0000238
90. Yousefi S, Perozzo R, Schmid I, Ziemiecki A, Schaffner T, Scapozza L, et al. Calpain-mediated cleavage of Atg5 switches autophagy to apoptosis. Nat Cell Biol (2006) 8:1124-32. doi:10.1038/ncb1482

Conflict of Interest Statement: The authors declare that the research was conducted in the absence of any commercial or financial relationships that could be construed as a potential conflict of interest.

Copyright (c) 2017 Miyazaki and Miyazaki. This is an open-access article distributed under the terms of the Creative Commons Attribution License (CC BY). The use, distribution or reproduction in other forums is permitted, provided the original author(s) or licensor are credited and that the original publication in this journal is cited, in accordance with accepted academic practice. No use, distribution or reproduction is permitted which does not comply with these terms. 